\title{
Synchronization of Distant Optical Clocks at the Femtosecond Level
}

\author{
Jean-Daniel Deschênes, ${ }^{*}$ Laura C. Sinclair, Fabrizio R. Giorgetta, William C. Swann, Esther Baumann, \\ Hugo Bergeron, Michael Cermak, Ian Coddington, and Nathan R. Newbury ${ }^{\dagger}$ \\ National Institute of Standards and Technology, 325 Broadway, Boulder, Colorado 80305, USA \\ (Received 11 December 2015; revised manuscript received 8 March 2016; published 11 May 2016)
}

\begin{abstract}
The use of optical clocks or oscillators in future ultraprecise navigation, gravitational sensing, coherent arrays, and relativity experiments will require time comparison and synchronization over terrestrial or satellite free-space links. Here, we demonstrate full unambiguous synchronization of two optical time scales across a free-space link. The time deviation between synchronized time scales is below $1 \mathrm{fs}$ over durations from 0.1 to $6500 \mathrm{~s}$, despite atmospheric turbulence and kilometer-scale path length variations. Over 2 days, the time wander is $40 \mathrm{fs}$ peak to peak. Our approach relies on the two-way reciprocity of a single-spatial-mode optical link, valid to below 225 attoseconds across a turbulent 4-km path. This femtosecond level of time-frequency transfer should enable optical networks using state-of-the-art optical clocks or oscillators.
\end{abstract}

DOI: 10.1103/PhysRevX.6.021016

Subject Areas: Atomic and Molecular Physics, Optics, Optoelectronics

\section{INTRODUCTION}

Optical clocks reach absolute accuracies approaching $10^{-18}[1-5]$ and optical oscillators (e.g., cavity-stabilized lasers) can provide subfemtosecond timing stability over seconds [6-10]. A physical network of optical time scales, derived from these clocks, could enable dramatic improvements in precision navigation and timing [5,11,12], phased sensor arrays, tests of special and general relativity [5,13-16], clock-based geodesy [5,17-19], and even future searches for dark matter [20]. In these applications, the local optical time scale would be either compared against or synchronized to a distant time scale via terrestrial or satellite free-space links. Existing rf-based techniques can support time transfer over free-space links but are limited to 10 - to 100-ps accuracy and $\sim 1$-ps stability, 100 to 1000 times worse than optical oscillators [21-23]. The highest performance if system to date is planned for the ACES mission and will support 300 -fs timing stability at 300 -s integration and $<6$-ps timing stability over days from ground to space $[24,25]$. Optical clocks or oscillators with femtosecond precision will eventually require analogous optical, rather than rf, time-frequency transfer techniques to realize their full potential. Indeed, optical time-frequency transfer over fiber optics can achieve frequency transfer at $10^{-18}$ fractional stability over $1840 \mathrm{~km}$ [26,27], time transfer at subpicosecond stability over distances up to

\footnotetext{
*jean-daniel.deschenes@gel.ulaval.ca nathan.newbury@nist.gov
}

Published by the American Physical Society under the terms of the Creative Commons Attribution 3.0 License. Further distribution of this work must maintain attribution to the author(s) and the published article's title, journal citation, and DOI.
$1000 \mathrm{~km} \mathrm{[28-30],} \mathrm{and} \mathrm{subfemtosecond} \mathrm{stability} \mathrm{over}$ several kilometers [31-34]. These fiber links are appropriate for connecting national laboratories, but to support the broader applications of clock networks that include mobile or temporary stations, free-space optical timing links will be essential [35-37].

The challenge with comparing and synchronizing the time between distant clocks arises from the finite speed of light. A direct comparison of their time inevitably includes an unknown and variable path delay in transmitting one clock signal to the other. In two-way rf comparisons, this problem is overcome by transmitting the time signals between clocks in each direction. Subtraction of the measured arrival times then yields the clock time offsets independent of the path delay-provided the path is reciprocal with equal delay in both directions. Reference [36] introduced an analogous two-way timefrequency transfer approach in the optical domain based on frequency combs. In that work, the goal was to enable frequency comparisons between remote oscillators after postprocessing, although the demonstration made use of a common optical oscillator. Here, we pursue the much more challenging problem of two-way time comparison between two distant optical time scales and, with active real-time feedback, their time synchronization. The ability to not just compare but to synchronize two distant clocks at the femtosecond level over the air can be a powerful tool but has significant complexity as it requires real-time measurements of the absolute time offset between clocks, real-time communication between sites, and real-time adjustment of the synchronized clock, all with femtosecond-level precision. Moreover, to achieve femtosecond time synchronization, this two-way approach must cancel variations in the path length between the distant sites to 

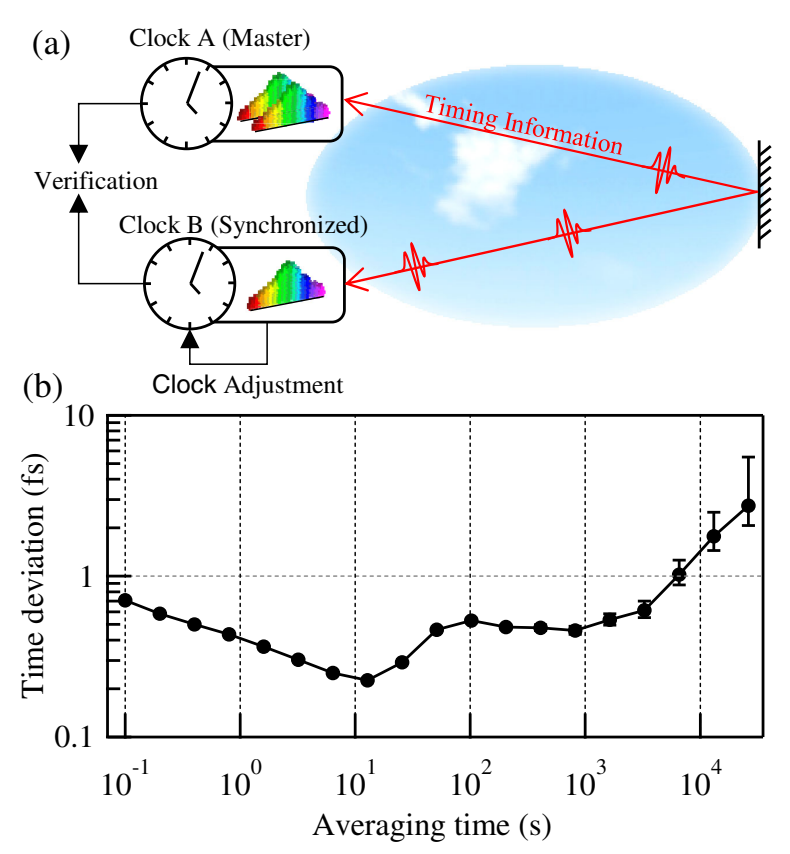

FIG. 1. (a) General synchronization concept. Time information is transmitted between sites across a turbulent air path. Real-time feedback is applied to the clock at site B to synchronize it with the clock at site A. A folded optical path allows for verification of the synchronization by a direct "out-of-loop" measurement. (b) Measured time deviation, or precision, between the time outputs while synchronized across a 4-km link, based on data acquired over 2 days as described in Sec. IV.

below $300 \mathrm{~nm}$ despite kilometer or longer paths through turbulent air.

Here, we show that optical two-way time transfer can indeed compare and synchronize two optical time scales to the femtosecond level and at an update rate of $2 \mathrm{kHz}$. The basic setup is sketched in Fig. 1(a). In this work, we construct two optical time scales based on independent cavity-stabilized lasers (i.e., optical oscillators). We show that these two time scales can be time synchronized with subfemtosecond stability from 0.1 to $6500 \mathrm{~s}$ [see Fig. 1(b)], dropping as low as 225 attoseconds at 10 -s averaging. A by-product of this synchronization is the syntonization (i.e., equalization) of the two clock frequencies with a relative instability reaching $2 \times 10^{-19}$. Over 2 days, the long-term wander of the time offset is 40 fs peak to peak, which is attributed to temperature-induced variations in the nonreciprocal fiber paths associated with the out-of-loop time verification and the coherent transceivers. This femtosecond-level performance is reached despite strong turbulence-induced fading and piston noise [38,39], variations of hundreds of picoseconds in the path delay from turbulence and weather, temporary misalignments of the link, and intentional variations of the path length from $1 \mathrm{~m}$ to $4 \mathrm{~km}$. At these levels, a network of optical clocks or oscillators will have a sensitivity 1000 times superior to analogous rf-based systems for timing, navigation, and sensing.

\section{SYNCHRONIZATION BETWEEN TWO DISTANT OPTICAL TIME SCALES USING TWO-WAY TIME TRANSFER}

A first requirement is to create two individual optical time scales at each site. The name notwithstanding, state-of-the-art atomic optical clocks are operated as frequency standards; they output an optical frequency from a laser stabilized to an optical cavity and atomic transition. Therefore, atomic optical clocks are always compared by their frequency ratios, typically via a frequency comb. To create a time scale instead, we phase lock a self-referenced frequency comb to a cavity-stabilized laser at each site. The optical pulses output by the frequency comb are then analogous to the "ticks" of a conventional clock. To generate a local time, we label the comb's optical pulses according to their arrival at a defined reference plane. Our goal is to synchronize the two clocks so that their pulses with the same time label arrive at their respective reference planes simultaneously.

We first review the simplest implementation of two-way time transfer, before discussing the modified optical twoway time transfer demonstrated here. Consider two clocks at separate sites A and B. Suppose site A transmits a pulse at its zero time to site $\mathrm{B}$. Its measured arrival time according to site B's clock is $\Delta \tau_{\mathrm{A} \rightarrow \mathrm{B}}=T_{\text {link }}+\Delta T_{\mathrm{AB}}$, where $T_{\text {link }}$ is the path delay and $\Delta T_{\mathrm{AB}}$ is the time offset between the clocks. Simultaneously, site B transmits a pulse at its assumed zero time in the opposite direction to site $\mathrm{A}$, where its arrival time is $\Delta \tau_{\mathrm{B} \rightarrow \mathrm{A}}=T_{\text {link }}-\Delta T_{\mathrm{AB}}$. Subtraction of these two arrival times yields the clock time offset, $\Delta T_{\mathrm{AB}}=\frac{1}{2}\left(\Delta \tau_{\mathrm{A} \rightarrow \mathrm{B}}-\Delta \tau_{\mathrm{B} \rightarrow \mathrm{A}}\right)$, that must be adjusted by a calibration constant $\tau_{\text {cal }}$ to account for time delays in the transceiver to a defined reference plane. Summation of the two arrival times provides $T_{\text {link }}$.

For our optical time scale, we cannot implement the simple two-way protocol discussed above with direct twoway exchange of the optical clock pulse trains because photodetection of the incoming pulses immediately introduces picosecond-level uncertainty, far in excess of the femtosecond timing jitter on the actual optical clock pulses. Instead, we implement linear optical sampling of the optical pulse trains [36]. Linear optical sampling requires the introduction of a third "transfer" comb that operates at an optical pulse repetition frequency offset by $\Delta f_{r}$ from both clocks' pulse train repetition frequency $f_{r}$. Heterodyne detection between this transfer comb and either clock comb yields an interferogram, or cross-correlation. From the peak location of the cross-correlation, we can infer the relative timing between pulse trains. Since the transfer comb's repetition frequency is offset by $\Delta f_{r}$, the cross-correlation repeats at this rate, yielding a relative timing measurement at an update rate of $\Delta f_{r}$ [36].

To combine this linear optical sampling approach with two-way transfer, we locate the transfer comb at site A. We then measure the two-way timing information across 
the link between the transfer comb and the clock comb at site $\mathrm{B}$, as well as the local timing information between the transfer comb and the clock comb at site A. Specifically, we use linear optical sampling to retrieve three quantities: (i) the time offset between the transmitted clock comb pulses from site $\mathrm{B}$ and the transfer comb pulses at site A, $\Delta \tau_{\mathrm{B} \rightarrow X}$, (ii) their analogous time offset at site $\mathrm{B}, \Delta \tau_{X \rightarrow \mathrm{B}}$, and (iii) the time offset between the transfer pulses and the comb pulses at site A, $\Delta \tau_{X \rightarrow \mathrm{A}}$. (Note that the subscript $X$ denotes the transfer comb.) As outlined in Appendix B, we can then derive a "master synchronization equation"- the analogy of the simple two-way time transfer equation given earlier-for the time offset between site A and site B as

$$
\begin{aligned}
\Delta T_{\mathrm{AB}}= & \frac{1}{2}\left(\Delta \tau_{\mathrm{B} \rightarrow X}-\Delta \tau_{X \rightarrow \mathrm{B}}\right)-\Delta \tau_{\mathrm{A} \rightarrow X}+\tau_{\mathrm{cal}} \\
& -\left(\frac{\Delta f_{r}}{2 f_{r}}\right)\left(T_{\text {link }}+\Delta t_{\mathrm{ADC}}\right)+\frac{\Delta n}{2 f_{r}},
\end{aligned}
$$

where $T_{\text {link }}$ is the time of flight across the link, $\Delta t_{\mathrm{ADC}}$ is the time offset between the analog-to-digital converters (ADCs) at the two sites, $\Delta n$ is an integer related to the pulse labeling, and $\tau_{\text {cal }}$ is a calibration offset that sets the location of the reference planes.

The first three terms of Eq. (1) comprise a generalized two-way time-transfer expression. There are two additional terms, one proportional to $\Delta f_{r}$ and one proportional to $\Delta n$. The latter simply accounts for the $1 /\left(2 f_{r}\right)$ ambiguity in the pulse labeling. The former is a small correction accounting for the mismatch in repetition rates between the transfer comb and clock combs. This mismatch is necessary for the linear optical sampling, but leads to an incomplete cancellation in the two-way comparison of both the path delay $T_{\text {link }}$ and the relative timing of the analog-to-digital converters used in the linear optical sampling $\Delta t_{\mathrm{ADC}}$. The term is small since it is proportional to $\Delta f_{r} /\left(2 f_{r}\right) \sim$ $1 / 200000$ but its inclusion is needed for correct time comparison and synchronization.

The frequency-comb-based measurements cannot provide a value for these last two terms. Instead, we require a "coarse" two-way time-transfer measurement that measures $\Delta t_{\mathrm{ADC}}$ and $T_{\text {link }}$ without ambiguity. To achieve 1-fs uncertainty in $\Delta T_{\mathrm{AB}}$, the uncertainty on these measurements must be below $2 f_{r} / \Delta f_{r} \times 1$ fs, and must be below $1 /\left(2 f_{r}\right)$ to resolve the integer $\Delta n$. In our system, the uncertainty of this coarse two-way time transfer needs to be below 200 ps.

Finally, calculation of Eq. (1) requires combining the two-way timing information measured at each site, which in turn requires rapid, real-time communication between them. Optical communication across a free-space link is well known to suffer from dropouts due to turbulence. Here, however, that problem is moot, as the optical communication channel uses the same single-mode spatial link as the comb light. Any turbulence-induced dropouts are correlated and, therefore, communication is available whenever the timing information is available. Once $\Delta T_{\mathrm{AB}}$ is calculated, its value can be used in a feedback loop to synchronize the clock at site B.

\section{EXPERIMENTAL IMPLEMENTATION}

Figure 2 shows a high-level view of the physical system. Sites A and B are connected via a free-space single-spatialmode optical link covering up to $4 \mathrm{~km}$. This link is folded by use of plane mirrors so that sites A and B are physically adjacent, enabling synchronization verification via an out-of-loop measurement of the time offset $\Delta T$, independent of the "in-loop" calculated time offset $\Delta T_{\mathrm{AB}}$. We discuss some important features of the experimental setup below with more details provided in Appendix C.

The optical time scale at each site is based on a cavitystabilized laser, which acts as an optical oscillator. The cavity-stabilized lasers for both sites are located in an environmentally stable laboratory that is $\sim 350 \mathrm{~m}$ from the main transceivers. A commercial cw fiber laser is locked to an optical cavity with a $\sim 1-\mathrm{Hz}$ linewidth and a typical environmentally induced frequency drift ranging from 0 to $10 \mathrm{~Hz} / \mathrm{s}$. The frequency of the cavity-stabilized laser is 195.297562 THz for site A and 195.297364 THz for site B. Two separate Doppler-canceled fiber links transport these frequencies to sites $\mathrm{A}$ and $\mathrm{B}$, located in a rooftop laboratory. To generate a time scale, at each site we phase lock a self-referenced "clock" frequency comb [40] to the cavity-stabilized laser to produce a $200.733423-\mathrm{MHz}$ pulse train at site $\mathrm{A}$ and a similar pulse train at site B. The detected pulses are used to clock a field programmable gate array (FPGA) controller that counts and labels each pulse with its arrival time at the selected reference plane, based on a given start time and known pulse repetition period. This conversion from an input optical frequency to a time requires that no phase slips occur in the conversion of the cavity-stabilized laser to the clock comb output; this is verified through monitoring of the phase locks of the cavitystabilized lasers, frequency combs, and Doppler-canceled links during synchronization. (However, when synchronized, any phase slips in the clock at site B are automatically compensated.) More generally, the cavity-stabilized laser could be steered to an atomic transition to provide an absolute time scale at a single master site, or at both sites, for example, in performing relativity experiments.

As described in the previous section, a third transfer comb is needed at the master site A. It is phase locked to site A's cavity-stabilized laser but with a pulse repetition rate that differs by $\Delta f_{r}=2.27 \mathrm{kHz}$ from the site A clock comb. The relative timing between the three comb pulse trains is then measured via linear optical sampling at three balanced detectors at an update rate of $\Delta f_{r}=2.27 \mathrm{kHz}$. From Nyquist sampling considerations, $\Delta f_{r}$ also sets a maximum transmitted comb optical bandwidth of $8 \mathrm{THz}$. Here, we filter the transmitted comb bandwidth to $16 \mathrm{~nm}$ 


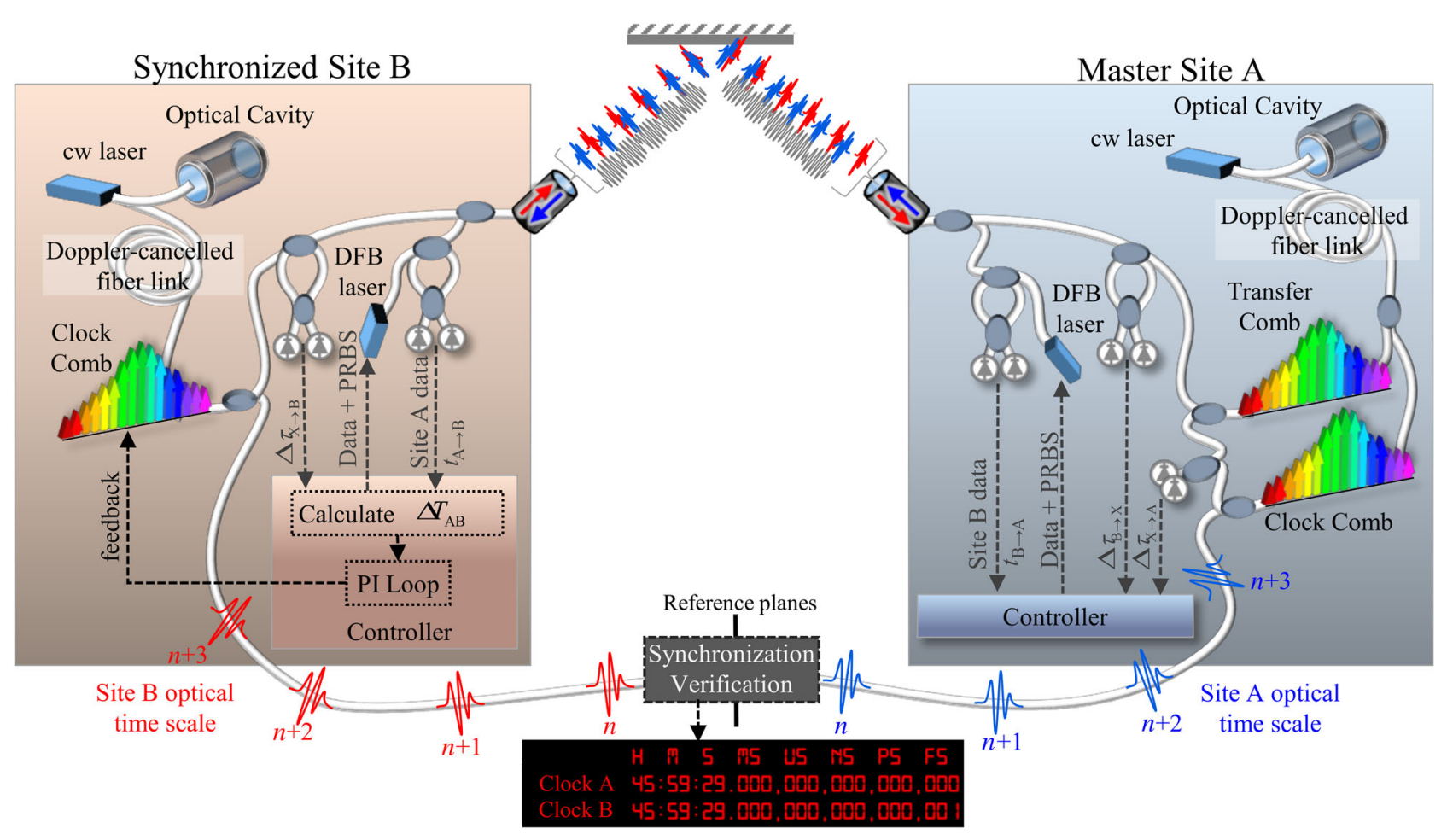

FIG. 2. Setup for the generation and synchronization of two optical time scales, one located at site A and one at site B, via optical twoway time-frequency transfer over a turbulent air path. The optical time scale from each site is defined by the arrival of pulses from the clock comb at a specified reference plane. For example, the $n$th pulse from each site arrives at the reference plane at the time shown in the lower display, while the $(n+1)$ st pulse arrives at a time $1 / f_{r}$ later. In the setup here, the two colocated clocks have a common reference plane to allow for out-of-loop synchronization verification. The timing and data exchange between the two sites occurs only over the free-space path shown in the upper part of the figure, and mimics a point-to-point link. DFB laser, distributed feedback laser and phase modulator; PI Loop, proportional-integral loop; symbols, see text.

(2 THz), centered at $1555 \mathrm{~nm}$. The comb power at the transmit aperture is $2.5 \mathrm{~mW}$.

We implement the coarse two-way time transfer, needed to establish the rightmost terms in Eq. (1), as in rf-based two-way time-frequency transfer [41], except that the timing signal is carried by rf phase modulation of a $\mathrm{cw}$ distributed feedback (DFB) laser with a pseudorandom binary sequence (PRBS). The PRBS-modulated DFB laser light and comb light are combined within the same singlemode fiber and launched via the same free-space optical terminals with tip-tilt control to compensate for turbulenceinduced beam wander. The PRBS-modulated light traverses the same single-mode spatial optical link as the two-way comb light and therefore measures the same path delay over the air. The detected signals measure the time difference between the ADC clock of site A at site $\mathrm{B} t_{\mathrm{A} \rightarrow \mathrm{B}}$ and the reciprocal quantity $t_{\mathrm{B} \rightarrow \mathrm{A}}$. Following the standard two-way approach described earlier, the sum and difference of these quantities yield unambiguous values of $T_{\text {link }}$ and $\Delta t_{\mathrm{ADC}}$ with a statistical uncertainty of $57 \mathrm{ps}$. This coarse two-way time transfer does see a different path delay than the comb light because of noncommon mode fiber-optic paths in the transceivers, but these differences are included in the calibration and their variations are suppressed by $\Delta f_{r} /\left(2 f_{r}\right) \approx 1 / 200000$ in Eq. (1) (see Appendix C).

The real-time optical communication is implemented across the free-space link using the same hardware as the coarse two-way time transfer, i.e., the phase modulation of the DFB laser light. The data communication is interposed between the two-way transmission of the PRBS signals so that they both occur within a single $1 / \Delta f_{r}$ interval. The controller at site B combines its local measurements with data from site $\mathrm{A}$ to calculate an in-loop time offset $\Delta T_{\mathrm{AB}}$ from Eq. (1), updated at $1 / \Delta f_{r}=0.5$-ms intervals. For real-time synchronization, $\Delta T_{\mathrm{AB}}$ is fed back via a proportional-integral loop filter to adjust the rf offset between the site B clock comb and its cavity-stabilized laser. Effectively, this feedback speeds up or slows down clock B to force $\Delta T_{\mathrm{AB}}$ to zero, thereby synchronizing the clocks. For loop stability considerations, the bandwidth of this feedback should be below $\sim \Delta f_{r} / 4=500 \mathrm{~Hz}$. Here, however, based on the free-running noise of the cavity-stabilized laser and measurement noise level on $\Delta T_{\mathrm{AB}}$, a $10-\mathrm{Hz}$ feedback bandwidth minimizes the residual timing jitter.

Before commencing the experiments, the system must be calibrated, which amounts to cross-calibrating the coarse 
two-way transfer with the comb-based measurement and selecting the phase delays of the remote clock so its reference plane coincides with the master clock. With this common reference plane, we can verify that pulses with the same time label do indeed arrive simultaneously. In an application, the sites would not be colocated and a common reference plane would be neither possible nor sensible. In that case, the remote transceiver would first need to be calibrated against the master transceiver and then moved, or, alternatively, a third portable system could be used to cross-calibrate the remote and master transceivers, depending on the requirements of the application (see Appendix C for details on the calibration).

\section{RESULTS}

As shown in Fig. 1, we verify the time synchronization by direct "out-of-loop" measurements of the time offset $\Delta T$ that are completely independent of the calculated "in-loop" value $\Delta T_{\mathrm{AB}}$.

The most sensitive measurement of $\Delta T$ is achieved by heterodyne detection between the two optical time scale outputs-i.e., the 200-MHz pulse trains from the combsat the common reference plane. To do this, the carrierenvelope offset frequency of the frequency comb at site B is purposefully offset relative to the comb at site $\mathrm{A}$ by $1 \mathrm{MHz}$. In this case, the heterodyne signal of comb pulses overlapping in time at the reference plane appears at $1 \mathrm{MHz}$ with an amplitude that depends on their time offset, as illustrated in Fig. 3(a). In the calibration of the remote transceiver, the common reference plane is set such that

(a)
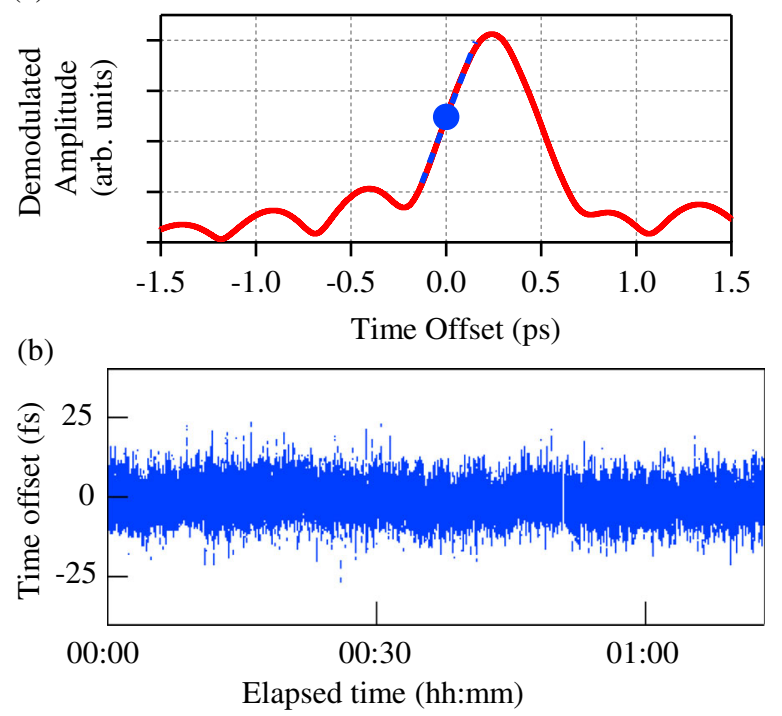

FIG. 3. (a) Heterodyne cross-correlation amplitude between the two clock pulse trains versus their time offset. The blue dashed line indicates the linear range of response. (b) Typical measured out-of-loop time offset $\Delta T$ over a $4-\mathrm{km}$ air path based on the heterodyne amplitude. While synchronized, the standard deviation is $2.4 \mathrm{fs}$. the response falls in the linear region, i.e., the blue dot in Fig. 3(a), and the amplitude gives a direct measure of the variation in the time offset. Figure 3(b) shows an example of the measured out-of-loop time offset over the 4-km link. Over the 1-h interval, the full standard deviation is $2.4 \mathrm{fs}$. The next section provides similar data over a longer time period and for varying path lengths.

These data alone do not verify that the time scales are unambiguously synchronized, i.e., that there are no $1 / f_{r}=$ 5-ns slips. Section IV C provides data on comparison of an optical pulse-per-second (PPS) output through direct photodetection. It also compares synchronous $1-\mathrm{Hz}$ pulse bursts through direct detection of spatial interference fringes between the optical pulses. For the latter, we are observing optical spatial interference between the $\sim 100$-fs optical pulses of two sources that are connected only via a 4-km free-space link.

\section{A. Synchronization over multiple days}

Figure 4 summarizes an experiment where sites A and B are synchronized for over $50 \mathrm{~h}$ across a $4-\mathrm{km}$ free-space
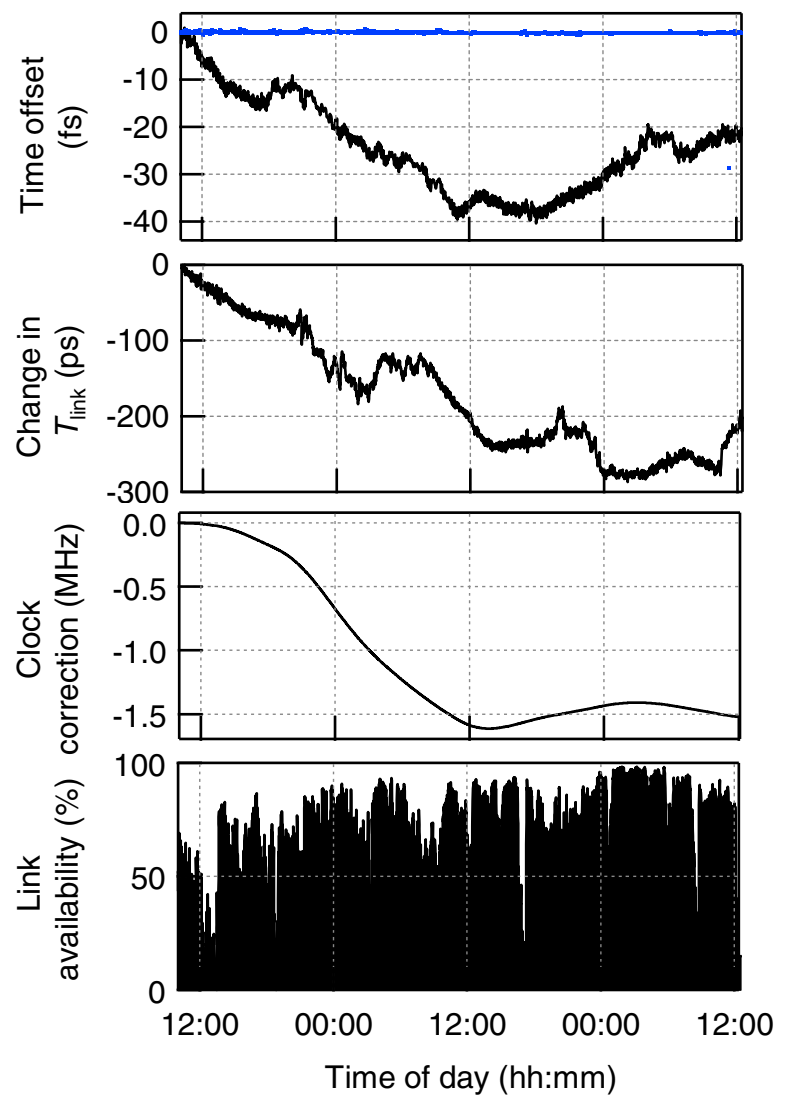

FIG. 4. Synchronization data across 4-km air path over a 50-h time period including, from top to bottom, the measured time offsets for both the out-of-loop $\Delta T$ (black trace) and the in-loop $\Delta T_{\mathrm{AB}}$ (blue trace), the change in time of flight $T_{\text {link }}$, the frequency correction applied to the time scale at site B to maintain synchronization, and the link availability. All data are filtered and down-sampled from the $0.5-\mathrm{ms}$ measurement period to $60 \mathrm{~s}$. 
link. The system runs without user intervention despite $4{ }^{\circ} \mathrm{C}$ rooftop laboratory temperature changes and ends with the arrival of a large snowstorm. (The system is able to operate through light snow and rain but not under heavy precipitation.)

The top panel plots the out-of-loop time offset as measured using the technique outlined in Fig. 3. These data are smoothed to $60 \mathrm{~s}$. [An expanded view of the unsmoothed performance over short time periods is given in Fig. 3(b).] The time-dependent offset is best analyzed by the timing deviation of these data, plotted in Fig. 1(b), which is the statistical uncertainty in the time offset as a function of averaging time [42]. From Fig. 1(b), this uncertainty is below 1 fs out to $6500 \mathrm{~s}(1.8 \mathrm{~h})$, reaching a minimum of 225 attoseconds for a 10-s average. Therefore, we infer that the single-spatial-mode link reciprocity over the $4-\mathrm{km}$ air path is verified to below $70 \mathrm{~nm}$ at 10 -s averaging and below $300 \mathrm{~nm}$ out to $6500 \mathrm{~s}$. Figure 4 shows that over the full 50-h measurement, the time offset exhibits a larger 40-fs peak-to-peak wander. This time wander does not reflect a breakdown in reciprocity over the free-space link since a shorted link exhibits the same behavior. Instead, it reflects a weak temperature dependence of the system to the $4{ }^{\circ} \mathrm{C}$ laboratory temperature variations. Specifically, we attribute most of this wander to temperature-driven path length variations in the $\sim 2 \mathrm{~m}$ of fiber that connects the two sites to the common reference plane and within the transceivers.

The second panel of Fig. 4 plots the variation of the time of flight $T_{\text {link }}$. (The average value of $T_{\text {link }}$ is $\sim 13 \mu \mathrm{s}$, corresponding to the $3942-\mathrm{m}$ path distance). $T_{\text {link }}$ varies by $290 \mathrm{ps}$ over the measurement period, corresponding to an $8.7-\mathrm{cm}$ variation in optical path. The variation is driven by turbulence and building motion on short periods and by atmospheric temperature changes on longer periods.

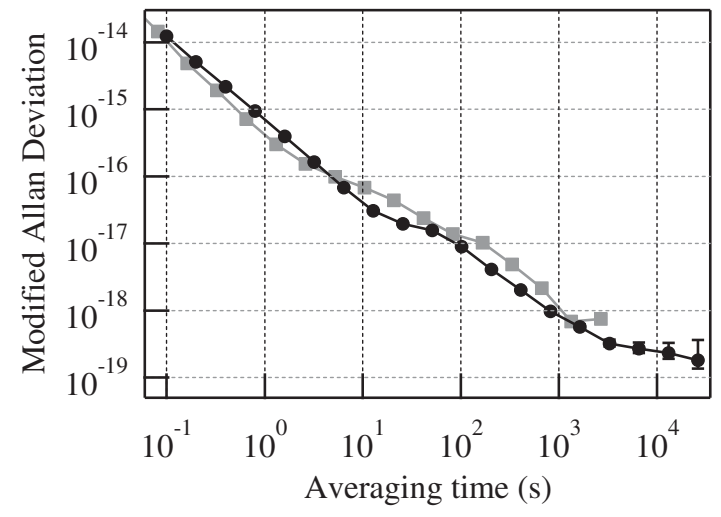

FIG. 5. Modified Allan deviation for the corresponding frequency transfer from site A to site B (black trace). The fractional frequency uncertainty reaches $2 \times 10^{-19}$. Up to $10^{3} \mathrm{~s}$, the uncertainty in the syntonized frequencies matches the frequency-comparison measurement of Ref. [36] over $2 \mathrm{~km}$ (gray trace) despite the additional complexity.
(Synchronization under $\mathrm{km}$-scale path variations is shown in the next section.)

The third panel of Fig. 4 plots the frequency correction that is applied to the 195.3-THz optical signal underlying the site $\mathrm{B}$ time scale. The effective time correction is given by the integral of this curve normalized by the optical oscillator frequency of $195.3 \mathrm{THz}$ and reaches $0.98 \mathrm{~ms}$ over the $50 \mathrm{~h}$, reflecting the time wander between the two freerunning cavity-stabilized lasers. One of the implicit by-products of full synchronization is full syntonization, or "frequency lock." The residual frequency uncertainty between the sites is given by the modified Allan deviation, which is simply the timing deviation of Fig. 1(b) multiplied by $\sqrt{3} / t_{\mathrm{av}}$, where $t_{\mathrm{av}}$ is the averaging time. As shown in Fig. 5, this Allan deviation is consistent with the earlier 2-km comparison measurement of Ref. [36], despite the additional complexity of full-time synchronization and longer distance. Moreover, it extends to longer averaging times reaching as low as $2 \times 10^{-19}$ beyond $10000 \mathrm{~s}$.

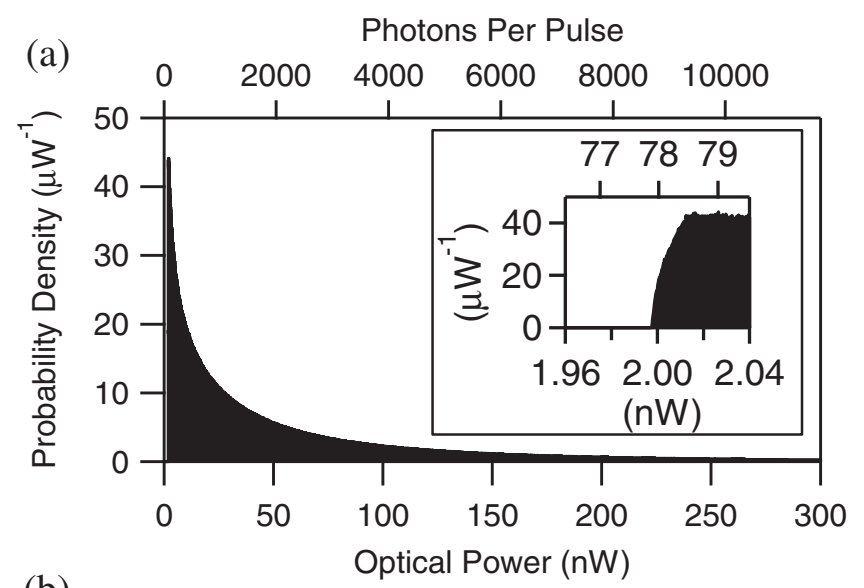

(b)

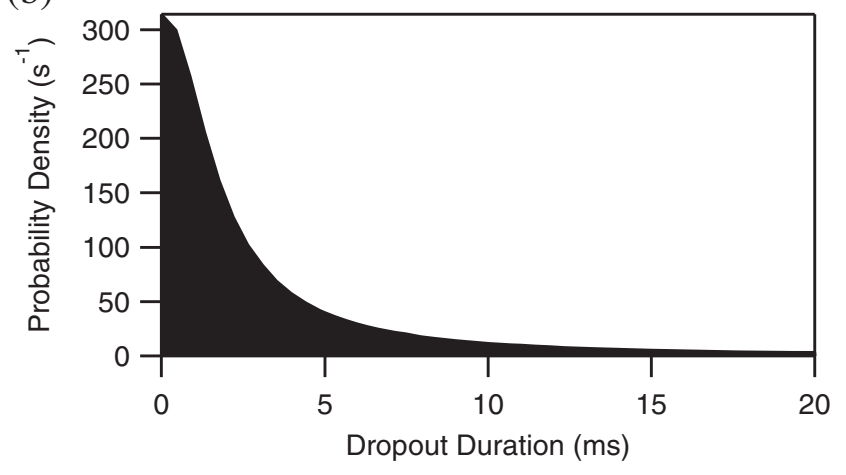

FIG. 6. Turbulence-induced power fluctuations and dropouts. (a) Probability density of received optical comb power for the $50 \mathrm{~h}$ of data shown in Fig. 4. Inset shows the $\sim 2-\mathrm{nW}$ threshold. (b) Probability density of dropouts versus duration. $90 \%$ of the dropouts are below $10 \mathrm{~ms}$. Longer durations are typically due to a disruption of the beam from physical objects, realignment, or heavy precipitation, rather than turbulence. The typical turbulence structure function is $C_{n}^{2} \approx 10^{-14} \mathrm{~m}^{-2 / 3}$ over the link. 
Atmospheric turbulence causes fluctuations in the received power. When it falls below the detection threshold, the link is unavailable and there is a "dropout." The bottom panel of Fig. 4 shows the percent of time per minute that the link is available. Figure 6(a) shows the distribution of received comb power given the $2.5-\mathrm{mW}$ launched power (well below the eye safe limit.) It varies from 0 to $\sim 200 \mathrm{nW}$, with a median value of $33 \mathrm{nW}$, compared to the detection threshold of $2 \mathrm{nW}$, or $\sim 78$ photons per pulse. When dropouts do occur, the synchronization is inactive and, therefore, these periods are excluded from the time offset data. However, these dropouts are typically below $10 \mathrm{~ms}$ in duration, as shown in Fig. 6(b), and the cavitystabilized lasers are well behaved so that the time offset at reacquisition is typically below 6 fs. For systems that require a continuous output, a Kalman filter could be implemented. This is especially critical for less-wellbehaved oscillators and long dropout durations. In general, the requirements on the free-running stability of the remote oscillator will depend on the dropout duration. In the case of very short dropouts, the Hz-level cavity-stabilized laser used here is excessive. We require only that the integral of

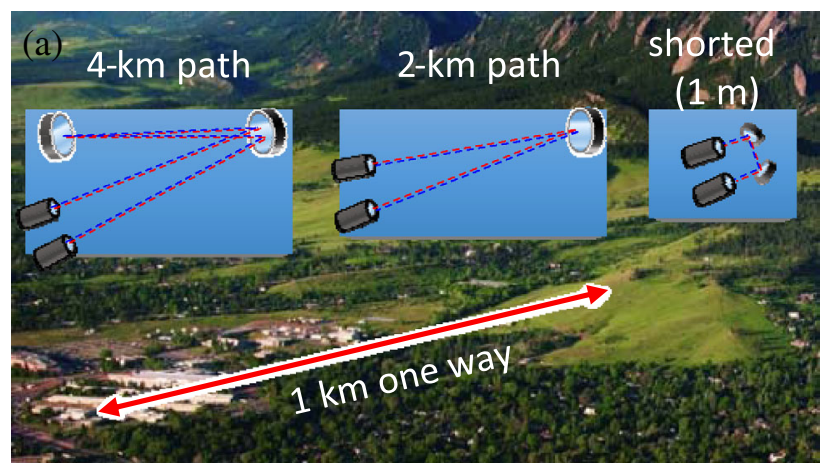

(b)

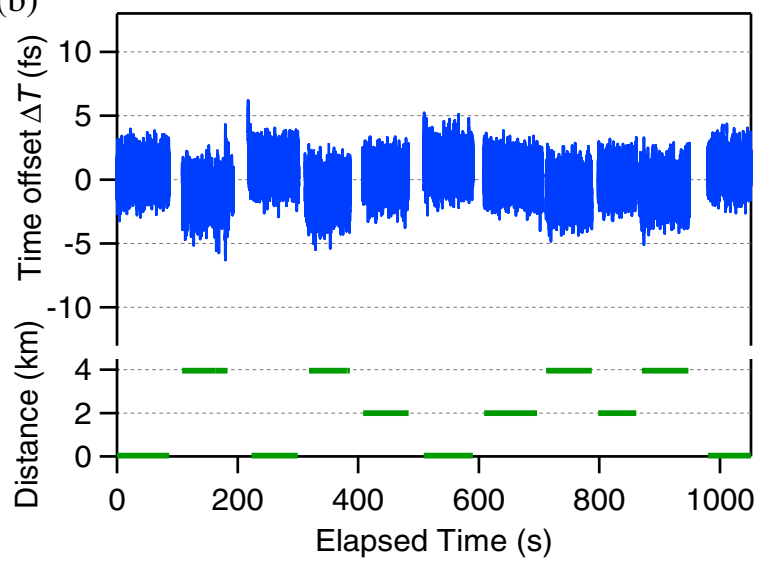

FIG. 7. (a) The link traverses the National Institute of Standards and Technology (NIST) campus over 1-m, 2-km, and 4-km distances with the latter achieved by a double pass between two flat mirrors. (b) The out-of-loop time offset $\Delta T$ (blue) as the link distance (green) is changed in real time. the remote oscillator's timing-jitter power spectral density, calculated from infinity to the inverse dropout duration, be below our system noise floor of $\sim 10$ fs or, more generally, the synchronization level needed for the application. In other words, since the active real-time synchronization suppresses any slower timing wander of the remote clock, it should be possible to operate the remote clock with a less stable and simpler local oscillator, at least for the case of short turbulence-induced dropouts.

\section{B. Synchronization maintained despite kilometer-scale length changes}

The synchronization is robust against large changes in link distance. In Fig. 7, the out-of-loop time offset $\Delta T$ is measured while the link distance is alternated between $1 \mathrm{~m}$, $2 \mathrm{~km}$, and $4 \mathrm{~km}$ by manually adjusting the folding mirrors, as indicated in Fig. 7(a). Each adjustment requires about 30 s. The system runs continuously during the link realignment, successfully resynchronizing within tens of milliseconds of reacquisition of the light across the link. The time offset shows a slow wander uncorrelated with distance that is similar to Fig. 4. In addition, there is a small $<2$-fs systematic time shift that is correlated with distance. We speculate that there are two effects that can cause this small time shift. First, any higher-order effects of atmospheric dispersion will cause distance-dependent time shifts. Second, any chromatic aberrations in the free-space telescopes can cause slight distance-dependent spectral filtering of the received comb pulses. This slight spectral filtering, combined with a chirp on the comb pulses, will lead to a slight time shift (in an effect that is very akin to Gordon-Haus jitter). Separate tests find negligible $(<1 \mathrm{fs})$ systematic shifts with received power.

\section{Optical pulse per second}

In conventional time systems, a rf PPS [24,28] provides unambiguous time markers. Here, we demonstrate femtosecond-level, unambiguous synchronization by generating analogous optical PPS signals. These optical PPS signals are easily generated by gating out a single pulse from the $200-\mathrm{MHz}$ optical pulse train. At each site, the optical pulse train is fiber coupled to a Mach-Zehnder amplitude modulator (MZM) that is driven from a gate pulse generated by the local FPGA controller. Since this FPGA controller tracks the time associated with each optical pulse, it can gate every 200 millionth pulse (where we define our time scales such that the comb repetition rates are exactly $200 \mathrm{MHz}$ ). These pulses still carry the precision and accuracy of the synchronized time scales as they still consist of 150-fs long optical pulses. To verify unambiguous timing, each gated pulse is photodetected and then their arrival is compared on a high-bandwidth oscilloscope. To verify synchronicity, the common reference plane must be shifted by adjusting $\tau_{\text {cal }}$ from that of 
(a)

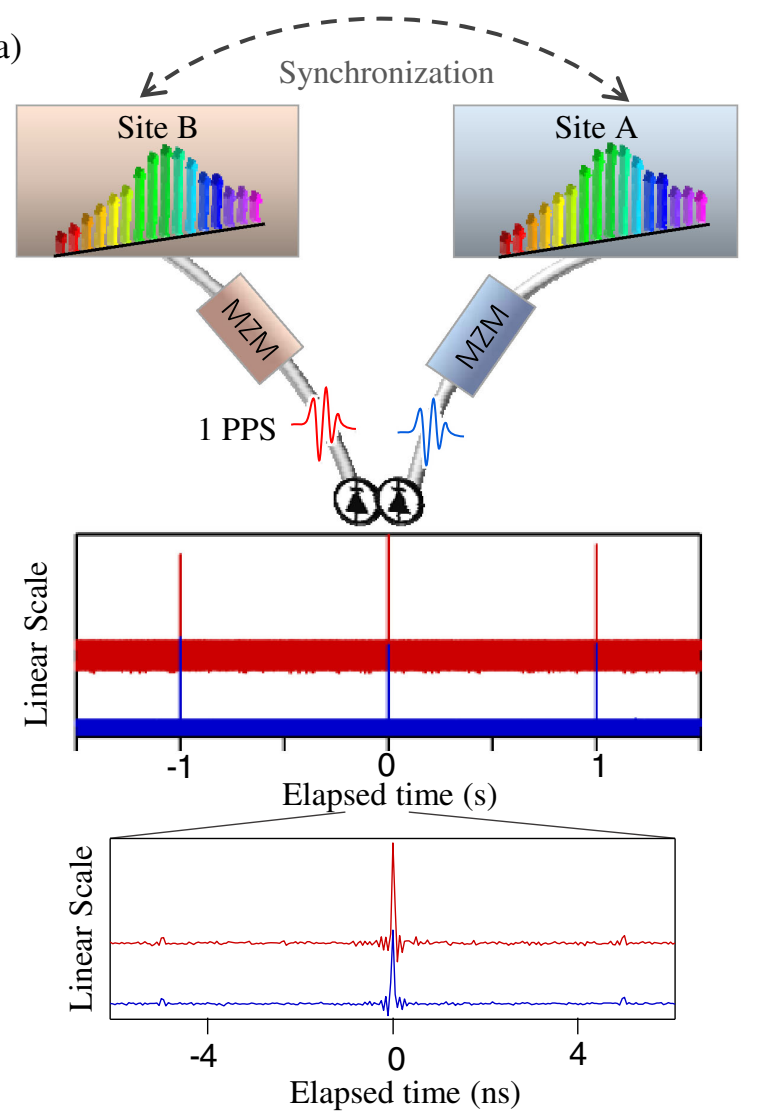

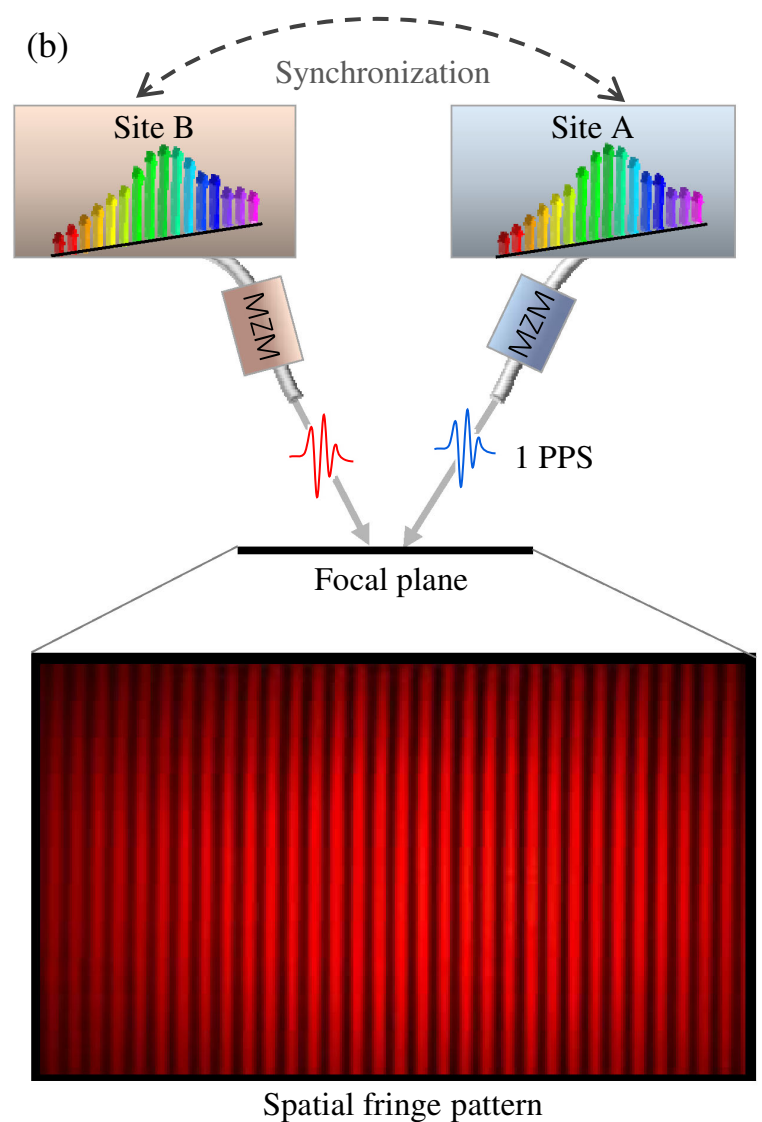

FIG. 8. Demonstration of synchronous optical pulse-per-second (PPS) outputs. (a) Synchronous optical PPS photodetection at 8-GHz bandwidth. (b) Optical interference between selected pulse bursts measured through the tilt interference pattern on a focal plane array. The strong interference demonstrates that the pulses arrive well within their correlation time of $\sim 300$ fs. (See also Video 1). MZM, Mach-Zehnder modulator.

Fig. 4 to compensate for relative delays between photodetection and the oscilloscopes. Figure 8(a) shows an example of synchronization of 1-PPS signals to below $\sim 100 \mathrm{ps}$, limited by the detector bandwidth. As with Fig. 7, synchronization is preserved across large path length variations.

These data illustrate that the timing is unambiguous, but the uncertainty is limited by the rf bandwidths. As a more sensitive demonstration, we can spatially interfere the optical PPS from the two time scales. To do this, we construct a spatial interference fringe pattern by coupling the two optical outputs into free space and combining them at a slight angle onto an InGaAs focal plane array. A single PPS pair provides insufficient photons across the focal plane array, so we increase the gate time to the MZM for a burst of pulses. Spatial interference fringes will be visible only when those bursts occur at the same time, and only when the pulses within the burst overlap in time to well within their $\sim 150$-fs duration. The presence of the high-contrast spatial interference pattern indicates unambiguous, femtosecond-level synchronization between sites.
Figure 8(b) shows such an interference pattern. Video 1 in Appendix A shows the appearance and disappearance of this spatial interference as synchronization is applied or disabled at site $\mathrm{B}$.

\section{DISCUSSION}

The results of the previous section demonstrate that the reciprocity of single-spatial-mode optical links is sufficient to support femtosecond synchronization of distant optical time scales. Moreover, it is possible to achieve this synchronization in a complex, but robust implementation that can operate for days, over turbulent paths, and over paths of very different lengths.

In the system here, the two time scales are synchronized relative to each other to below $1 \mathrm{fs}$ for up to $1.8 \mathrm{~h}$. They are not stabilized to an absolute established time scale, although the master site A could be in principle. This low residual timing is nevertheless useful for a distributed passive or active sensing system or for navigation. In particular, synchronization of distant optical time scales 
could enable future high-precision navigation or timing networks, e.g., an optically based global navigation satellite system, by synchronizing compact optical oscillators to a few, more complex and larger master optical atomic clocks. The increased accuracy and precision can then yield improved location information, although any rf-based space-to-ground timing distribution would still be limited by atmospheric effects. For other applications, it might be necessary to include an atomic clock at the master site A. For clock-based geodesy or relativity experiments, full atomic clocks are needed at each site for time comparisons. In that case, the residual timing noise associated with any comparison (or synchronization) between sites will be well below the absolute noise of the time scales.

Systematic time offsets with distance are below a few femtoseconds at $4 \mathrm{~km}$, and no systematics are observed with variations in received optical power. There are, however, two important systematics. First, there will be temperature-induced path length changes in nonreciprocal optical paths either within the transceivers or in the out-ofloop verification. These effects can be suppressed by appropriate experimental design and by temperature control, down to tens of femtoseconds, as shown here. Second, the width of the optical pulses is 100-fs to 1-ps long; the exact definition of the time associated with these pulses depends on how the end user "reads out" the arrival time of the pulse center at the reference plane, which will necessarily depend on the application. Again, this systematic will be on the order of tens of femtoseconds.

rf-based two-way time-frequency transfer is much more developed and can operate over much longer rangesincluding ground to space - and to moving platforms [24]. Here, our 4-km path is horizontal and therefore suffers equivalent turbulence to a longer vertical ground-tosatellite path, but longer distance operation will have higher transmission loss and path delay $T_{\text {link }}$. The higher transmission loss will need to be offset by a reduced detection threshold, higher transmit powers, and improved free-space terminals, possibly including adaptive optics. The longer path delay can potentially cause a breakdown in the reciprocity condition, which assumes a "fixed" turbulence over the two-way measurement time of $1 / \Delta f_{r}$. For $T_{\text {link }} \gg 1 / \Delta f_{r}$, the short-term turbulence-induced piston noise [39] is not completely negligible, but the long-term piston noise should nevertheless be canceled via the twoway approach.

Moving platforms present at least two additional problems: point-ahead issues and Doppler shifts. For transverse motion between platforms, the "point-ahead" effect causes the two signals to traverse slightly different optical paths and, therefore, causes a breakdown in reciprocity. As with the impact of a longer path delay, this effect is strongest in a ground-to-space scenario. These effects have been analyzed recently by Wolf and co-workers [43], who found an increase in the timing noise over short times below a few seconds but excellent two-way cancellation over longer times. The impact of Doppler shifts will require further study. In principle, this two-way method should be valid even with the effects of Doppler shifts. However, the exact implementation here is not Doppler insensitive because of dispersion within the transceivers, and future work is needed to optimize the system for insensitivity to Doppler shifts and to quantify any performance penalties.

\section{CONCLUSIONS}

We demonstrate real-time time transfer and synchronization between remote optical time scales using two-way exchange of optical pulses over a reciprocal free-space link. We verify subfemtosecond time synchronization out to $1.8 \mathrm{~h}$. The long-term wander over 2 days is 40 fs peak to peak, dominated by measurement uncertainty in the out-ofloop verification. The system is operated over a turbulent 4-km free-space path, but we find no fundamental limitations associated with distance. The single-mode free-space path is fully reciprocal to within our measurement uncertainty, which reaches $70 \mathrm{~nm}$ at 10-s averaging. Provided sufficient received power is available (here, equal to 78 photons per pulse), this approach should be scalable to much longer paths. The performance is 1000 times superior to rf-based methods and should enable future networks of optical clocks or oscillators that are synchronized in real time with subfemtosecond stability.

\section{ACKNOWLEDGMENTS}

We acknowledge funding from NIST and under the DARPA DSO PULSE program as well as helpful comments from Prem Kumar. We also acknowledge helpful comments from Marla Dowell, David Hume, and Andrew Ludlow, and technical assistance from Chris Cromer and Joe Thompson.

\section{APPENDIX A: VIDEO}

Video 1 is provided to show the spatial intensity of the overlapping PPS pulses from sites A and B, recorded with an InGaAs focal plane array as in Fig. 8 during operation over a 4-km link. Spatial fringes appear and disappear as the synchronization is activated, deactivated, and reactivated.

\section{APPENDIX B: DERIVATION OF THE MASTER SYNCHRONIZATION EQUATION}

We outline a derivation of the master synchronization equation. There are several factors that complicate the derivation. First, the derivation necessarily requires writing the time output of a clock versus time. In the timefrequency community, this notational challenge is 


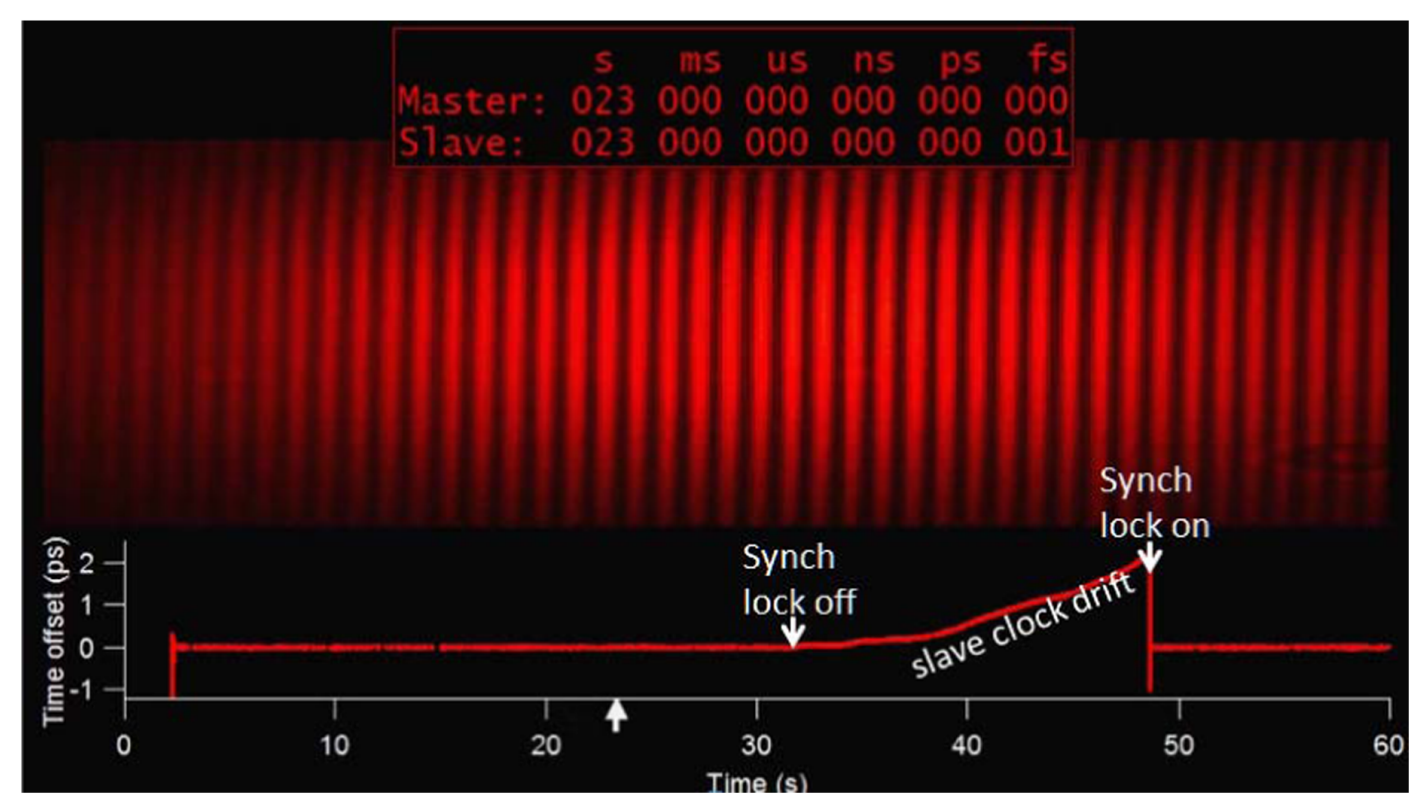

VIDEO 1. Video demonstrating synchronous optical PPS outputs. (See also Fig. 8.) The video shows the strong interference during synchronization and the lack of an interference pattern when clock B is free-running. The strong interference during synchronization verifies that the pulses arrive well within their correlation time of $\sim 300 \mathrm{fs}$.

sometimes handled by introducing the " $x$ " variable for the clock time to write $x(t)$ or by viewing the clock output as a phase. The phase description is a useful one for this system as well, but is not pursued here. Rather, we write the time marker from the $n$th comb pulse as $T(n) \equiv n f_{r}^{-1}+\tau(t)$ in terms of its repetition rate $f_{r}$ and slowly varying time offset $\tau$, as a function of some "absolute" time $t$ (which does not appear in the final synchronization equation). Second, there are multiple ambiguities that appear as "modulo" operations versus the comb pulse period $1 / f_{r}$ and the interferogram repetition period $1 / \Delta f_{r}$. These ambiguities must be appropriately handled for any absolute time comparisons between clocks.

The comb at site A produces a pulse train that is coherent with its cavity-stabilized cw laser,

$$
E_{\mathrm{A}}(t)=e^{i \phi_{\mathrm{A}}} \sum_{n_{\mathrm{A}}} e^{-i n_{\mathrm{A}} \theta_{0}} A_{\mathrm{A}}\left(t-n_{\mathrm{A}} f_{r, \mathrm{~A}}^{-1}-\tau_{\mathrm{A}}\right),
$$

where $\phi_{A}$ is an arbitrary phase, $\theta_{0}$ is the carrier-envelope offset phase, $n_{\mathrm{A}}$ is a comb pulse index, $f_{r, \mathrm{~A}}$ is the repetition rate, and $\tau_{\mathrm{A}}$ is the time offset. In this form, it is clear the comb outputs pulses whose arrival time provides the time markers $T_{\mathrm{A}}\left(n_{\mathrm{A}}\right) \equiv n_{\mathrm{A}} f_{r, \mathrm{~A}}^{-1}+\tau_{\mathrm{A}}(t)$. Alternatively, the comb can be written as

$$
E_{\mathrm{A}}(t)=e^{i \phi_{A}} \sum_{k_{\mathrm{A}}} \tilde{A}_{k, \mathrm{~A}} e^{-i 2 \pi\left(k_{\mathrm{A}} f_{r, \mathrm{~A}}+f_{0, \mathrm{~A}}\right)\left(t-\tau_{\mathrm{A}}\right)},
$$

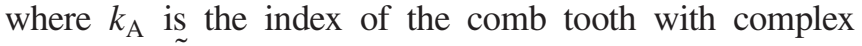
amplitude $\tilde{A}_{k, \mathrm{~A}}$ at frequency $k_{\mathrm{A}} f_{r, \mathrm{~A}}+f_{0, \mathrm{~A}}$, where the carrier-envelope offset frequency $f_{0, \mathrm{~A}} \equiv(2 \pi)^{-1} \theta_{0} f_{r, \mathrm{~A}}$. This second, equivalent form is useful in deriving the interferogram produced by the product of two combs below.

The other two combs have exactly the same form with variable subscripts " $B$ " and " $X$ " instead of "A." We assume that the repetition rates are perfect; i.e., we attribute all of the time-varying clock error to $\tau$, which becomes a slowly varying function of time $\tau(t)$. (In this case, slowly varying means slow on the time scale of $1 / \Delta f_{r}$.) We then denote the repetition rates as $f_{r, \mathrm{~A}}=f_{r, \mathrm{~B}} \equiv f_{r}$ and the difference in repetition rate between the site $\mathrm{A}$ comb and transfer combs as $\Delta f_{r} \equiv f_{r, X}-f_{r, \mathrm{~A}}$.

Linear optical sampling, as in Ref. [36], allows us to achieve femtosecond-level precision by recording the heterodyne signal between the pulse train from a remote comb and local oscillator comb, i.e., their cross-correlation that appears as an interferogram. Interferograms are detected in three locations (given by the balanced detectors in Fig. 2). These interferograms repeat with a period $1 / \Delta f_{r}$ as the comb pulses walk through each other. The interferogram voltages are digitized by the local analog-to-digital converter, which is clocked at the local (site A or B) comb repetition rate. The interferograms digitized at site A have ADC sample times of $t_{\mathrm{A}}=n_{\mathrm{A}} f_{r}^{-1}+t_{0, \mathrm{~A}}$, where $t_{0, \mathrm{~A}}$ includes the time delay in detecting the comb pulse and any delays within the ADC itself. The interferogram digitized at site $\mathrm{B}$ has sample time of $t_{\mathrm{B}}=n_{\mathrm{B}} f_{r}^{-1}+t_{0, \mathrm{~B}}$. From the product of the comb electric fields [Eq. (B2)] and including a low-pass filter, the digitized interferograms are the series 


$$
\begin{aligned}
& V_{\mathrm{A} \rightarrow X}\left(t_{\mathrm{A}}\right) \propto \sum_{p} I_{\mathrm{A} \rightarrow X}\left(t_{\mathrm{A}}+p \Delta f_{r}^{-1}+\frac{1}{\Delta f_{r}}\left[f_{r}\left(\tau_{\mathrm{A}}+\delta \tau_{\mathrm{A}}\right)-\left(f_{r}+\Delta f_{r}\right)\left(\tau_{X}+\delta \tau_{X}\right)\right]\right), \\
& V_{\mathrm{B} \rightarrow X}\left(t_{\mathrm{A}}\right) \propto \sum_{q} I_{\mathrm{B} \rightarrow X}\left(t_{\mathrm{A}}+q \Delta f_{r}^{-1}+\frac{1}{\Delta f_{r}}\left[f_{r}\left(\tau_{\mathrm{B}}+\delta \tau_{\mathrm{B}}+T_{\text {link }}\right)-\left(f_{r}+\Delta f_{r}\right)\left(\tau_{X}+\delta \tau_{X}^{\prime}\right)\right]\right), \\
& V_{X \rightarrow \mathrm{B}}\left(t_{\mathrm{B}}\right) \propto \sum_{s} I_{X \rightarrow \mathrm{B}}\left(t_{\mathrm{B}}-s \Delta f_{r}^{-1}-\frac{1}{\Delta f_{r}}\left[f_{r}\left(\tau_{\mathrm{B}}+\delta \tau_{\mathrm{B}}^{\prime}\right)-\left(f_{r}+\Delta f_{r}\right)\left(\tau_{X}+\delta \tau_{X}^{\prime \prime}+T_{\text {link }}\right)\right]\right)
\end{aligned}
$$

after dropping any phase terms, where $p, q$, and $s$ are integers that essentially label consecutive interferograms of length $1 / \Delta f_{r}, I_{\mathrm{A} \rightarrow X}$ is the cross-correlation of the subscripted pulse envelopes again with an analogous definition for the two other series. For the first two interferograms, the transfer comb (comb $X$ ) serves as the local oscillator, while for the third term, comb B does, which causes the sign difference in the envelope terms. The $\delta \tau$ values represent the extra time delay in the transceivers associated with the comb reaching the relevant balanced detector. $T_{\text {link }}$ is the time delay over the reciprocal single-mode path.

We extract the peak location of each interferogram after matched-filter processing to improve the signal-to-noise ratio. We then scale these peak locations by $\Delta f_{r} / f_{r}$ to find

$$
\begin{aligned}
& \Delta \tau_{\mathrm{A} \rightarrow X}(p)=-p f_{r}^{-1}-\left(\tau_{\mathrm{A}}+\delta \tau_{\mathrm{A}}\right)+\frac{\left(f_{r}+\Delta f_{r}\right)}{f_{r}}\left(\tau_{X}+\delta \tau_{X}\right), \\
& \Delta \tau_{\mathrm{B} \rightarrow X}(q)=-q f_{r}^{-1}-\left(\tau_{\mathrm{B}}+\delta \tau_{\mathrm{B}}+T_{\text {link }}\right)+\frac{\left(f_{r}+\Delta f_{r}\right)}{f_{r}}\left(\tau_{X}+\delta \tau_{X}^{\prime}\right), \\
& \Delta \tau_{X \rightarrow \mathrm{B}}(s)=s f_{r}^{-1}+\left(\tau_{\mathrm{B}}+\delta \tau_{\mathrm{B}}^{\prime}\right)-\frac{\left(f_{r}+\Delta f_{r}\right)}{f_{r}}\left(\tau_{X}+\delta \tau_{X}^{\prime \prime}+T_{\text {link }}\right)-\frac{\Delta f_{r}}{f_{r}} \Delta t_{\mathrm{ADC}},
\end{aligned}
$$

where $\Delta \tau_{X \rightarrow B}$ includes the time offset between the two sites' $\mathrm{ADCs}$ as $\Delta t_{\mathrm{ADC}} \equiv t_{\mathrm{A}}-t_{\mathrm{B}}=\Delta n f_{r}^{-1}+t_{0, \mathrm{~A}}-t_{0, \mathrm{~B}}$, where $\Delta n \equiv n_{\mathrm{A}}-n_{\mathrm{B}}$. Based on the coarse two-way time transfer, we align the $p, q$, and $s$ integers to compare the interferograms that are closest in time (to within $1 / \Delta f_{r}$ ). The linear combination

$$
\begin{aligned}
& \frac{1}{2}\left[\Delta \tau_{\mathrm{B} \rightarrow X}(p)-\Delta \tau_{X \rightarrow \mathrm{B}}(p)-2 \Delta \tau_{\mathrm{A} \rightarrow X}(p)\right] \\
& \quad=\left(\tau_{\mathrm{A}}-\tau_{\mathrm{B}}\right)+\left(\frac{\Delta f_{r}}{2 f_{r}}\right)\left(T_{\text {link }}+\Delta t_{\mathrm{ADC}}\right)+\delta \tau^{\prime}
\end{aligned}
$$

yields the slowly varying time offset between the sites (the first term) with additional contributions from the imperfect cancellation of the slowly varying link delay and ADC time offsets. The last term is the appropriate linear combination of the various $\delta \tau$ terms in Eq. (B4), which are assumed constant.

We are interested in the time offset at the reference plane, which is defined as $\Delta T_{\mathrm{AB}}=\left(\tau_{\mathrm{A}}+\delta \tau_{\text {ref, } \mathrm{A}}\right)-$ $\left(\tau_{\mathrm{B}}+\delta \tau_{\text {ref, } \mathrm{B}}\right)$, where $\delta \tau_{\text {ref,A }}$ is the fixed delay of the site A pulses to the site A reference plane and $\delta \tau_{\text {ref,B }}$ is similarly defined. (Here, the two have the same reference plane so that we can verify synchronization.) We therefore rearrange Eq. (B5) to find

$$
\begin{aligned}
\Delta T_{\mathrm{AB}}(p)= & \frac{1}{2}\left[\Delta \tau_{\mathrm{B} \rightarrow X}(p)-\Delta \tau_{X \rightarrow \mathrm{B}}(p)\right]-\Delta \tau_{\mathrm{A} \rightarrow X}(p) \\
& +\tau_{\text {cal }}-\left(\frac{\Delta f_{r}}{2 f_{r}}\right)\left(T_{\text {link }}+\Delta t_{\mathrm{ADC}}\right)+\frac{\Delta n}{2 f_{r}},
\end{aligned}
$$

or Eq. (1) in the main text, where $\tau_{\text {cal }}=\delta \tau_{\text {ref,A }}-\delta \tau_{\text {ref,B }}-\delta \tau^{\prime}$. $\tau_{\text {cal }}$ must be measured via a calibration step. Variations in $\delta \tau$ can lead to systematic time wander, as observed over the 2-day measurement. In this equation, we also explicitly add a term proportional to $\Delta n=n_{\mathrm{A}}-n_{\mathrm{B}}$ associated with the index of the pulses.

\section{APPENDIX C: METHODS}

Frequency combs.-The self-referenced frequency combs follow the design of Ref. [40]. The 972 920th mode of comb A is phase locked to the site A optical cavity, while the 972909 th mode of the transfer comb, comb $X$, is phase locked to the same cavity. This leads to values of $f_{r}=$ $200.733423 \mathrm{MHz}$ and $\Delta f_{r}=2.27 \mathrm{kHz}$. The 972919 th mode of comb B is phase locked to the site B optical cavity, so that its repetition frequency is close to $f_{r}$ before the synchronization loop is closed. The combs, as well as the other fiber optics associated with the linear optical sampling and communication or PRBS system, are enclosed in small temperature-controlled aluminum boxes within a larger transceiver box, which is loosely temperature controlled.

Coarse two-way time transfer. -The coarse two-way time transfer is accomplished via a phase-modulated $\mathrm{cW}$ DFB laser at $1536.2 \mathrm{~nm}$. At each site, the local FPGA controller applies a phase modulation to a local DFB laser via an external phase modulator. To enable coherent detection, the two DFB lasers are frequency locked to an offset of $150 \mathrm{MHz}$ by measurement of the incoming 

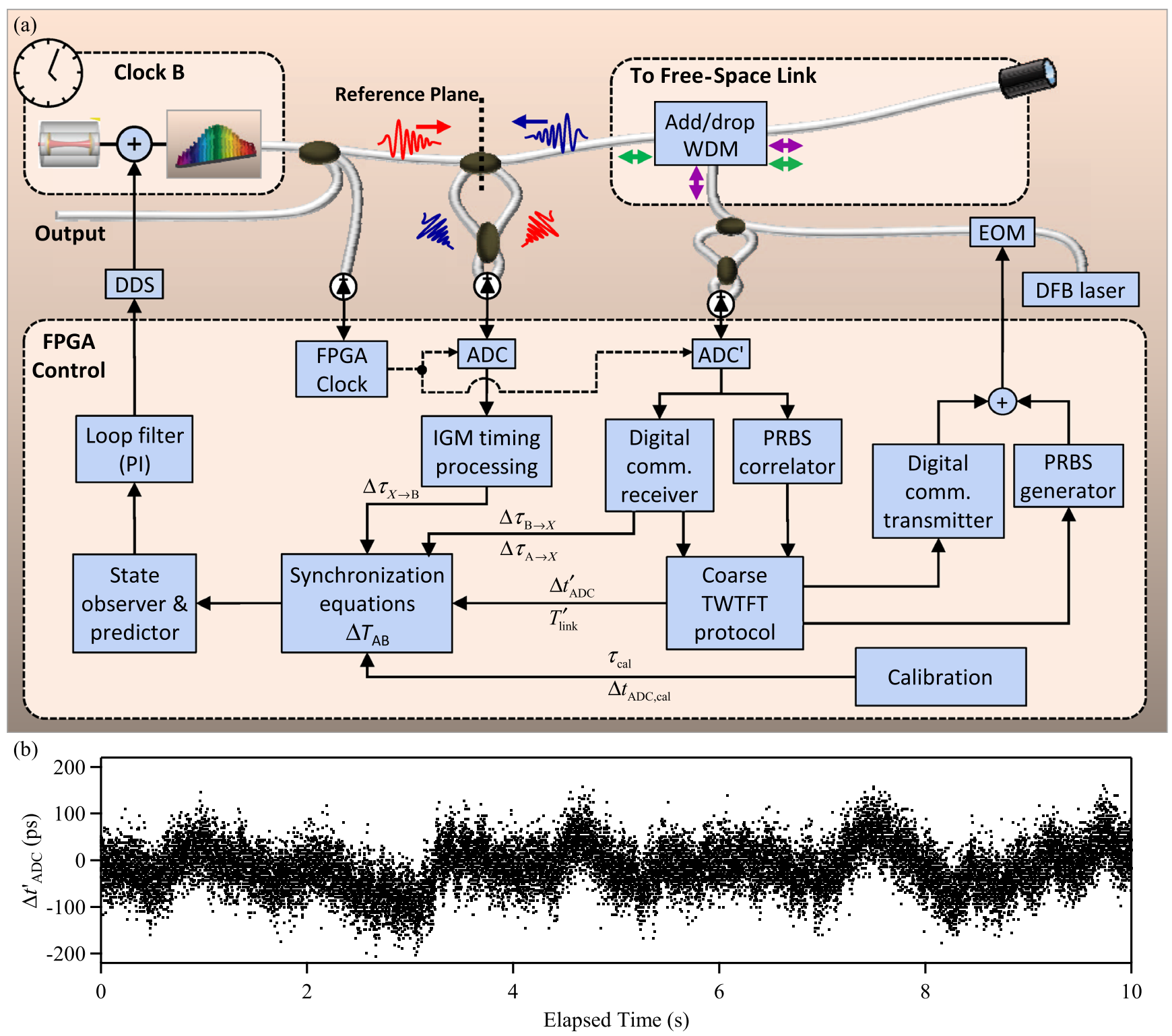

FIG. 9. (a) Detailed schematic of system on site B that defines the various quantities relevant to the calibration and to the calculation of the master synchronization equation. See text for details. DDS, direct digital synthesizer; PRBS, pseudorandom binary sequence; ADC, analog-to-digital converter for the linear optical sampling between comb pulse trains; ADC', analog-to-digital converter for the coarse two-way time transfer via the PRBS signals; DFB, distributed feedback laser; EOM, Electro-optic phase modulator; PI, proportionalintegral loop filter; WDM, wavelength division multiplexer; IGM, interferograms. TWTFT, two-way time-frequency transfer. (b) Time synchronization between the ADC clocks of the remote and master sites with a standard deviation of below 57 ps. (Longer duration data have the same standard deviation.)

light from site A at site B. At each site, the DFB laser light is combined with the comb light through a wavelength division multiplexer (WDM). To implement the coarse twoway time transfer [41], site A first transmits a 80- $\mu$ s-long ( $\sim 800$ chips) Manchester-coded pseudorandom binary sequence phase-modulated laser signal at 100-ns chip length ( $\sim 10 \mathrm{Mb} / \mathrm{s}$ signaling rate). When this is completed, site B transmits its own PRBS phase-modulated light signal across the link. Both sites use coherent detection to demodulate both the incoming and outgoing PRBS signal and time stamp the departure and arrival of the PRBS signals according to the local ADC time base. (Note that we time stamp the transmission of the PRBS signal with the same ADC used to time stamp the arrival of the PRBS from the opposite terminal to avoid any additional time offsets inherent in the PRBS generation.) From the appropriate linear combination of the four time stamps, we obtain $\Delta t_{\mathrm{ADC}}^{\prime}$ and $T_{\text {link }}^{\prime}$, where the prime indicates these values are associated with the coarse two-way transfer link delay and its ADC (see Fig. 9). Because of this exchange of unique 
time stamps (64 bits), this measurement has, for all practical matters, infinite ambiguity range $\left(5 \mathrm{~ns} \times 2^{64}\right)$.

Communication link.-For real-time communication between the sites, the same hardware is used as the coarse two-way time transfer. The coherent phase-modulated light operates in half-duplex mode using Manchester-encoded binary phase shift keying at $10 \mathrm{Mbps}$ and a protocol tolerant to link dropouts with low $(10 \mu \mathrm{s})$ latency. Data integrity is ensured by a simple 10-bit cyclic redundancy error-detection code in each packet. The full bidirectional data and coarse two-way time transfer require $350 \mu \mathrm{s}$.

Calibration.-As with any two-way time transfer, we need to calibrate the transceivers to remove differential time delays between the two-way timing measurements and the clock reference planes. In general, the master and remote sites would not be colocated, and either the remote transceiver would need to be precalibrated against the master transceiver and then relocated, or a third "transfer" transceiver would be needed. Here, the master and remote transceivers are colocated, so these steps are not needed, but the basic prescription described below also applies to this more general case.

There are several important factors related to the calibration for the femtosecond-level synchronization demonstrated here. First, as noted previously, it is critical to use the correct master synchronization equation that includes the added terms proportional to $\Delta f_{r} / f_{r}$. Second, from this equation, it is clear that we require not only the expected calibration factor $\tau_{\text {cal }}$, but also the additional real-time values for $T_{\text {link }}$ and $\Delta t_{\mathrm{ADC}}$. These latter two values refer to the two-way comb-based time transfer, but they are provided by the coarse rf-based two-way time transfer. As a result, we require an added cross-calibration step between the two time-transfer systems. Third, femtosecond synchronization requires a system design that minimizes all differential fiber-optic paths between the clock output and the two-way timing measurements. The optical design is given in Ref. [44]. As one example, the clock comb's output is combined with its cavity-stabilized laser at a physical point that is adjacent to the cross-correlation or interferogram timing measurement to avoid differential fiber path lengths, rather than in a location separated by meters of fiber.

We implement the calibration using the same hardware as for the heterodyne synchronization verification measurement. In this way, we calibrate the reference plane of the remote clock to coincide with the master clock's reference plane (so that we can verify synchronization during the actual measurements). We first cross-calibrate the coarse two-way time transfer against the comb-based two-way transfer. This step is independent of the reference plane. As discussed above, the coarse two-way time transfer measures the time offset between the analog-to-digital converters of the two transceivers to support the master synchronization equation. However, as shown in Fig. 9(a), the coarse two-way time transfer actually measures the time offset between the primed ADC of the remote site and its counterpart at the master site, $\Delta t_{\mathrm{ADC}}^{\prime}$, rather than measuring the needed time offset, $\Delta t_{\mathrm{ADC}}$, between the unprimed ADC in Fig. 9 and its counterpart; the two are not equal due to clock distribution within the ADC board. We calibrate their difference, $\Delta t_{\mathrm{ADC} \text {,cal }}=\Delta t_{\mathrm{ADC}}^{\prime}-\Delta t_{\mathrm{ADC}}$, by recording the value $\Delta t_{\mathrm{ADC}}^{\prime}$ when $\Delta T$ crosses zero for an unsynchronized system. Once calibrated, $\Delta t_{\mathrm{ADC}}=\Delta t_{\mathrm{ADC}}^{\prime}-\Delta t_{\mathrm{ADC} \text {,cal }}$ must remain constant to within $\sim 200 \mathrm{ps}$ for subfemtosecond uncertainty in the master synchronization equation [because of the factor of $\Delta f_{r} / f_{r}$ in Eq. (1)]. Figure 9(b) shows values for $\Delta t^{\prime}{ }_{\mathrm{ADC}}$ with a standard deviation of $57 \mathrm{ps}$, well within the $200 \mathrm{ps}$ requirement.

In a very similar vein, the coarse two-way time transfer measures a two-way path delay $T_{\text {link }}^{\prime}$ that is not identical to the two-way path delay of the comb pulses $T_{\text {link }}$, because of differing lengths of fiber before the wavelength multiplexing in Fig. 9. (The free-space portion of the path is identical.) The difference between these two path delays could be treated in an identical fashion to $\Delta t_{\mathrm{ADC}}$ as described above. However, for simplicity, we instead include the factor $\left(\Delta f_{r} / f_{r}\right)\left(T_{\text {link }}-T_{\text {link }}^{\prime}\right)$ within the calibration of $\tau_{\text {cal }}$, which also includes the appropriate linear combination of all the fiber-optic path delays as discussed below Eq. (B6).

Once the cross-calibration is completed, the remote clock is synchronized and the value of $\tau_{\text {cal }}$ adjusted until $\Delta T=0$. As implemented, the value of $\tau_{\text {cal }}$ corresponds to a phase delay applied to the direct digital synthesizer (DDS) driven by the FPGA controller of Fig. 9(a). This value is then stored for future use.

After this final calibration, the two sites are fully synchronized; i.e., not only do the two clocks "tick" at the same rate, they read the same time at the reference plane. The link can be interrupted by a long dropout, by manual unlocking, by breaking the phase lock of the transfer comb or remote comb, by breaking the phase lock of the DFB lasers, etc., and the full synchronization is always regained using the same calibration values. Since $\tau_{\text {cal }}$ is a "number" within the FPGA controller, it can be adjusted at will by the user to shift the reference plane using stored calibration values, for example, between that needed for Figs. 4 and 8.

Finally, it is critical that the steering of the remote clock does not introduce additional timing noise. This steering is accomplished by adjusting the offset frequency between the relevant comb tooth and the cavity-stabilized laser. Thus, any timing noise on the DDS in Fig. 9(a) is suppressed by a factor of $\sim f_{r} / 195 \mathrm{THz} \sim 10^{-6}$.

Free-space link.-The combined comb and communication or PRBS light is launched across the 4-km path from single-mode fiber at the input of a free-space terminal. The free-space optical terminals use tip-tilt control to compensate for beam wander due to turbulence and building sway. A 5-mW beacon laser at either 1532.7 or $1542.9 \mathrm{~nm}$, well 
separated from the other wavelengths, is polarization multiplexed with the comb and communication or PRBS light. The combined beams are then expanded in an offaxis, reflective parabolic telescope and launched over free space. At the receiver, the beam is collected by an identical terminal, and a dichroic then directs the beacon laser light to a quadrant detector, while the comb and communication or PRBS light are coupled into single-mode, polarizationmaintaining fiber, which is then connected to the combbased transceiver. The signals from the quadrant detector on each side are fed into an analog feedback system that controls the tip or tilt through an $x-y$ galvanometric mirror pair, thereby centering the beacon laser and maximizing the comb and communication or PRBS light coupled into the single-mode fiber.

[1] C. W. Chou, D. B. Hume, J. C. J. Koelemeij, D. J. Wineland, and T. Rosenband, Frequency Comparison of Two HighAccuracy $\mathrm{Al}^{+}$Optical Clocks, Phys. Rev. Lett. 104, 070802 (2010).

[2] N. Hinkley, J. A. Sherman, N. B. Phillips, M. Schioppo, N. D. Lemke, K. Beloy, M. Pizzocaro, C. W. Oates, and A. D. Ludlow, An Atomic Clock with $10^{-18}$ Instability, Science 341, 1215 (2013).

[3] B. J. Bloom, T. L. Nicholson, J. R. Williams, S. L. Campbell, M. Bishof, X. Zhang, W. Zhang, S. L. Bromley, and J. Ye, An Optical Lattice Clock with Accuracy and Stability at the $10^{-18}$ Level, Nature (London) 506, 71 (2014).

[4] I. Ushijima, M. Takamoto, M. Das, T. Ohkubo, and H. Katori, Cryogenic Optical Lattice Clocks, Nat. Photonics 9 , 185 (2015).

[5] A. D. Ludlow, M. M. Boyd, J. Ye, E. Peik, and P. O. Schmidt, Optical Atomic Clocks, Rev. Mod. Phys. 87, 637 (2015).

[6] S. A. Webster, M. Oxborrow, S. Pugla, J. Millo, and P. Gill, Thermal-Noise-Limited Optical Cavity, Phys. Rev. A 77, 033847 (2008).

[7] J. Millo, D. V. Magalhães, C. Mandache, Y. Le Coq, E. M. L. English, P. G. Westergaard, J. Lodewyck, S. Bize, P. Lemonde, and G. Santarelli, Ultrastable Lasers Based on Vibration Insensitive Cavities, Phys. Rev. A 79, 053829 (2009).

[8] D. R. Leibrandt, M. J. Thorpe, M. Notcutt, R. E. Drullinger, T. Rosenband, and J.C. Bergquist, Spherical Reference Cavities for Frequency Stabilization of Lasers in NonLaboratory Environments, Opt. Express 19, 3471 (2011).

[9] Y. Y. Jiang, A. D. Ludlow, N. D. Lemke, R. W. Fox, J. A. Sherman, L.-S. Ma, and C. W. Oates, Making Optical Atomic Clocks More Stable with $10^{-16}$-Level Laser Stabilization, Nat. Photonics 5, 158 (2011).

[10] T. Kessler, C. Hagemann, C. Grebing, T. Legero, U. Sterr, F. Riehle, M. J. Martin, L. Chen, and J. Ye, A Sub-40-mHzLinewidth Laser Based on a Silicon Single-Crystal Optical Cavity, Nat. Photonics 6, 687 (2012).

[11] P. Gill, When Should We Change the Definition of the Second?, Phil. Trans. R. Soc. A 369, 4109 (2011).
[12] P. Kómár, E. M. Kessler, M. Bishof, L. Jiang, A. S. Sørensen, J. Ye, and M. D. Lukin, A Quantum Network of Clocks, Nat. Phys. 10, 582 (2014).

[13] L. Cacciapuoti and C. Salomon, Space Clocks and Fundamental Tests: The ACES Experiment, Eur. Phys. J. Spec. Top. 172, 57 (2009).

[14] J. Müller, M. Soffel, and S. Klioner, Geodesy and Relativity, J. Geodes. 82, 133 (2008).

[15] P. Wolf et al., Quantum Physics Exploring Gravity in the Outer Solar System: The SAGAS Project, Exp. Astron. 23, 651 (2009).

[16] S. Schiller et al., Einstein Gravity Explorer-A MediumClass Fundamental Physics Mission, Exp. Astron. 23, 573 (2009).

[17] D. Kleppner, Time Too Good to Be True, Phys. Today 59, No. 3, 10 (2006).

[18] C. W. Chou, D. B. Hume, T. Rosenband, and D. J. Wineland, Optical Clocks and Relativity, Science 329, 1630 (2010).

[19] A. Yamaguchi, M. Fujieda, M. Kumagai, H. Hachisu, S. Nagano, Y. Li, T. Ido, T. Takano, M. Takamoto, and H. Katori, Direct Comparison of Distant Optical Lattice Clocks at the $10^{-16}$ Uncertainty, Appl. Phys. Express 4, 082203 (2011).

[20] A. Derevianko and M. Pospelov, Hunting for Topological Dark Matter with Atomic Clocks, Nat. Phys. 10, 933 (2014).

[21] A. Bauch, Time and Frequency Comparisons Using Radiofrequency Signals from Satellites, C.R. Phys. 16, 471 (2015).

[22] M. Fujieda, D. Piester, T. Gotoh, J. Becker, M. Aida, and A. Bauch, Carrier-Phase Two-Way Satellite Frequency Transfer over a Very Long Baseline, Metrologia 51, 253 (2014).

[23] D. Piester, M. Rost, M. Fujieda, T. Feldmann, and A. Bauch, Remote Atomic Clock Synchronization via Satellites and Optical Fibers, Adv. Radio Sci. 9, 1 (2011).

[24] P. Delva, F. Meynadier, C. Le Poncin-Lafitte, P. Laurent, and P. Wolf, in Proceedings of the European Frequency and Time Forum, EFTF 2012 (European Frequency and Time Forum, Gothenburg, Sweden, 2012), pp. 28-35.

[25] P. Laurent, D. Massonnet, L. Cacciapuoti, and C. Salomon, The ACES/PHARAO Space Mission, C.R. Phys. 16, 540 (2015).

[26] S. Droste, F. Ozimek, T. Udem, K. Predehl, T. W. Hänsch, H. Schnatz, G. Grosche, and R. Holzwarth, OpticalFrequency Transfer over a Single-Span $1840 \mathrm{~km}$ Fiber Link, Phys. Rev. Lett. 111, 110801 (2013).

[27] A. Bercy, F. Stefani, O. Lopez, C. Chardonnet, P.-E. Pottie, and A. Amy-Klein, Two-Way Optical Frequency Comparisons at $5 \times 10^{-21}$ Relative Stability over 100-km Telecommunication Network Fibers, Phys. Rev. A 90, 061802 (2014).

[28] M. Rost, D. Piester, W. Yang, T. Feldmann, T. Wübbena, and A. Bauch, Time Transfer through Optical Fibres over a Distance of $73 \mathrm{~km}$ with an Uncertainty below $100 \mathrm{ps}$, Metrologia 49, 772 (2012).

[29] Ł. Śliwczyński, P. Krehlik, A. Czubla, Ł. Buczek, and M. Lipiński, Dissemination of Time and RF Frequency via a Stabilized Fibre Optic Link over a Distance of $420 \mathrm{~km}$, Metrologia 50, 133 (2013).

[30] O. Lopez, A. Kanj, P.-E. Pottie, D. Rovera, J. Achkar, C. Chardonnet, A. Amy-Klein, and G. Santarelli, Simultaneous 
Remote Transfer of Accurate Timing and Optical Frequency over a Public Fiber Network, Appl. Phys. B 110, 3 (2013).

[31] J. Kim, J. A. Cox, J. Chen, and F. X. Kärtner, Drift-Free Femtosecond Timing Synchronization of Remote Optical and Microwave Sources, Nat. Photonics 2, 733 (2008).

[32] M. Xin, K. Şafak, M. Y. Peng, P. T. Callahan, and F. X. Kärtner, One-Femtosecond, Long-Term Stable Remote Laser Synchronization over a 3.5-km Fiber Link, Opt. Express 22, 14904 (2014).

[33] J.-F. Cliche and B. Shillue, Precision Timing Control for Radioastronomy: Maintaining Femtosecond Synchronization in the Atacama Large Millimeter Array, IEEE Control Syst. Mag. 26, 19 (2006).

[34] B. Ning, S. Y. Zhang, D. Hou, J. T. Wu, Z. B. Li, and J. Y. Zhao, High-Precision Distribution of Highly Stable Optical Pulse Trains with $8.8 \times 10^{-19}$ Instability, Sci. Rep. 4, 5109 (2014).

[35] K. Djerroud, O. Acef, A. Clairon, P. Lemonde, C. N. Man, E. Samain, and P. Wolf, Coherent Optical Link through the Turbulent Atmosphere, Opt. Lett. 35, 1479 (2010).

[36] F. R. Giorgetta, W. C. Swann, L. C. Sinclair, E. Baumann, I. Coddington, and N. R. Newbury, Optical Two-Way Time and Frequency Transfer over Free Space, Nat. Photonics 7, 434 (2013).

[37] E. Samain, P. Vrancken, P. Guillemot, P. Fridelance, and P. Exertier, Time Transfer by Laser Link (T2L2): Characterization and Calibration of the Flight Instrument, Metrologia 51, 503 (2014).
[38] L. C. Andrews and R. L. Phillips, Laser Beam Propagation through Random Media, Laser Beam Propagation through Random Media, 2nd ed. (SPIE, Bellingham, WA, 2005).

[39] L. C. Sinclair, F. R. Giorgetta, W. C. Swann, E. Baumann, I. Coddington, and N. R. Newbury, Optical Phase Noise from Atmospheric Fluctuations and Its Impact on Optical TimeFrequency Transfer, Phys. Rev. A 89, 023805 (2014).

[40] L. C. Sinclair, J.-D. Deschênes, L. Sonderhouse, W. C. Swann, I. H. Khader, E. Baumann, N. R. Newbury, and I. Coddington, Invited Article: A Compact Optically Coherent Fiber Frequency Comb, Rev. Sci. Instrum. 86, 081301 (2015).

[41] D. Kirchner, Two-Way Time Transfer via Communication Satellites, Proc. IEEE 79, 983 (1991).

[42] IEEE Standard Definitions of Physical Quantities for Fundamental Frequency, and Time Metrology-Random Instabilities, IEEE Std 1139-1999 1 (1999).

[43] C. Robert, J.-M. Conan, and P. Wolf, Impact of turbulence on high-precision ground-satellite frequency transfer with two-way coherent optical links, Phys. Rev. A 93, 033860 (2016).

[44] L. C. Sinclair, W. C. Swann, J.-D. Deschênes, H. Bergeron, F. R. Giorgetta, E. Baumann, M. Cermak, I. Coddington, and N. R. Newbury, in Slow Light, Fast Light, and OptoAtomic Precision Metrology, SPIE Proceedings Vol IX (SPIE-International Society for Optical Engineering, Bellingham, WA, 2016), p. 976308. 